\title{
Über das System Zinn und Jod.
}

\author{
Von \\ H. S. VAN KLOOSTER. \\ Mit 2 Figuren im Text.
}

Obwohl die älteren Untersuchungen über die Verbindungen von Zinn und Jod die Existenz von zwei Jodiden, das Stannojodid $\left(\mathrm{SnJ}_{2}\right)$ und das Stannijodid ( $\mathrm{SnJ}_{4}$ ) sichergestellt haben, so sind doch die Angaben über die Entstehung und die Existenzbedingungen dieser Verbindungen, namentlich des $\mathrm{Sn} J_{2}$ derart verwirrt und einander widersprechend, daß eine Neubearbeitung dieser Frage auf Grund des Schmelzdiagrammes nicht ohne Interesse schien. Ich habe deshalb das System Zinn.Jod experimentell untersucht, gleichfalls in Zusammenhang mit einer in dem hiesigen Laboratorium geplanten Untersuchung der Jodide der Kohlenstoffgruppe, die sich bei früheren Untersuchungen dieser Art anschließen soll. Beim Niederschreiben der Resultate erhielt ich Kenntnis einer Arbeit von W. ReINders und S. DE LANGE, ${ }^{1}$ worin dasselbe Problem behandelt wird. Wiewohl die Ergebnisse dieser Untersuchung sich sehr gut mit den hier veröffentlichten Beobachtungen decken, so erachte ich es indessen nicht für überfüssig, die erhaltenen Resultate kurz zusammenzufassen, um so mehr weil sie die Angaben der genannten Autoren in glücklicher Weise ergänzen, speziell in bezug auf die Darstellung und Charakterisierung des Dijodids.

Eine Besprechung und Kritik der früheren Untersuchungen kann hier unterbleiben, unter Verweisung auf die genannte Arbeit.

Als Ausgangsmaterialien wurden verwendet reines doppelt sublimiertes Jod (Schmp. 113.5 ) und Zinn in Barren (Schmp. 232 ${ }^{\circ}$ ), beide von C. A. F. Kahlbaum bezogen.

Das Zinn wurde, zwecks besserer Einführung in die Schmelzröbren von schwer schmelzbarem Duraxglas, vor dem Gebrauch in dünne Streifen geschnitten. Ferner fand ein reines, umkristallisiertes Präparat von $\mathrm{SnJ}_{4}$ Verwendung. Ein als $\mathrm{SnJ}_{2}$ bezeichnetes

1 W. Reinders u. S. de Lanae, Versl. v. d. Kon. Ak. v. Wetensch. 1912, p. 333-341. Siebe auch die vorangehende Abhandlung. 
Kahlbaumsches Produkt, das wahrscheinlich auf nassem Wege erhalten war, und den KorkverschluB unter Entwickelung saurer Dämpfe (Jodwasserstoff') stark angegriffen hatte, war für den beabsichtigten Zweck unbrauchbar. Die Mischungen, deren Zusammensetzung zwischen $\mathrm{J}$ und $\mathrm{SnJ} \mathrm{J}_{4}$ liegt, wurden durch Zusammenschmelzen dieser beiden Bestandteile in offenen Röhren erhalten. Durch vorsichtige Erwärmung, ein wenig über den Schmelzpunkt, gelingt es den Verlust auf ein Minimum zu beschränken. Außerdem wurde der Verlust, der niemals mehr als $0.05 \mathrm{~g}$ betrug (auf $10 \mathrm{~g}$ Substanz), jedesmal in Rechnung gebracht. Die Mischungen, deren Gebalt an Jod zwischen 0 und 80 Atom- $\%$ liegt, wurden alle aus leicht ersichtlichen Gründen in geschlossenen Röhren hergestellt und thermisch untersucht. Zwecks Einführung des Thermoelements aus Silberkonstantan (geeicht mittels siedendem Wasser, schmelzendem Zinn und Cadmium) war ein enges Glasröhrchen eingeschmolzen, das bis nahe an den Boden des Gefäßes reichte, um dem Thermoelement eine zentrale Lage in der Mitte der Schmelzen zu sichern. Das Zusammenschmelzen geschah jedesmal in einem horizontalen eisernen Zylinder, der in einem Gasöfchen erhitzt wurde und der mittels eines Gasmotors fortwährend auf und nieder bewegt wurde. Die Abküblung wurde in einem Nickelofen nach LoRENz ausgeführt.

Eine Zusammenfassung meiner thermischen Beobachtungen gibt die folgende Tabelle 1 und das Diagramm (Fig. 1). Zum Vergleich sind die von ReINDERS und DE LANGe erhaltenen Werte gleichfalls eingetragen und die bezüglichen Punkte im Diagramm mit Kreisen verzeichnet.

Die mit einem Sternchen $\left({ }^{*}\right)$ bezeichneten Versuche beziehen sich auf unvollständige Gleichgewichtseinstellungen in der Schmelze. Ich komme darauf noch näher zurück.

Es ist aus der Tabelle ersichtlich, daB die Angaben von ReINDERS und DE LANGE für das Teildiagramm von 100-80 Atom- $\%$ Jod ausführlicher sind als die meinigen, nur fehlen die Zahlen für die eutektische Mischung. Die Lage des Eutektikums wird angegeben bei 12.06 Atom. $\%$ Jod und $79.6^{\circ}$, während meine Zahlen $12 \%$ bzw. $76^{\circ}$ ergeben. Der Schmelzpunkt des Tetrajodids ist praktisch derselbe (Reinders und DE Lange $143.5^{\circ}$, EMich $143^{\circ}$, จ. KL. 143\%). Mischbarkeit in festem Zustand ist soweit ersichtlich nicht vorhanden; auch BeckManN, der in jüngster Zeit ${ }^{1}$ die Gefrier-

1 Z. anorg. Chem. 77 (1912), 200. 
Tabelle 1.

\begin{tabular}{|c|c|c|c|c|c|c|c|c|c|}
\hline \multirow{2}{*}{ 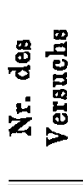 } & \multicolumn{2}{|c|}{$\begin{array}{l}\text { Zusammen- } \\
\text { setzung der } \\
\text { Schmelze in }\end{array}$} & \multirow{2}{*}{ 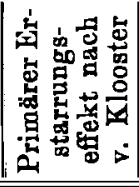 } & \multirow{2}{*}{ 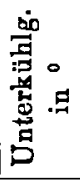 } & \multirow{2}{*}{ 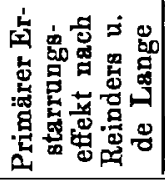 } & \multirow{2}{*}{ 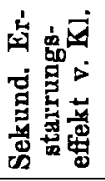 } & \multirow{2}{*}{ 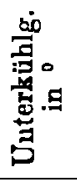 } & \multirow{2}{*}{ 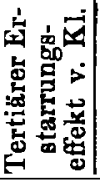 } & \multirow{2}{*}{ 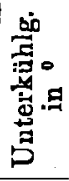 } \\
\hline & $\begin{array}{l}\text { Atom- } \\
\% \text { Jod }\end{array}$ & $\begin{array}{l}\text { Gew.- } \\
\% \text { Jod }\end{array}$ & & & & & & & \\
\hline 1 & 100 & 100 & 113.5 & - & 113.2 & & - & & \\
\hline 2 & 98.6 & 98.7 & 111.5 & - & & 72 & 0.5 & & \\
\hline & 98 & & & & 109 & & & & \\
\hline & 96 & & & & 104.7 & & & & \\
\hline & 93.95 & & & & 99.7 & & & & \\
\hline & 91.9 & & & & 94.7 & & & & \\
\hline 3. & 90.7 & 91.2 & 90 & - & & 76 & - & & \\
\hline & 89.9 & & & & 87.6 & & & & \\
\hline & 88.9 & & & & 83 & & & & \\
\hline 4 & 88.65 & 89.3 & 79 & - & & 76 & 1 & & \\
\hline & 87.9 & & & & 79.6 & & & & \\
\hline & 86.95 & & & & 83.5 & & & & \\
\hline & 85.95 & & & & 89.8 & & & & \\
\hline 5 & 84.8 & 85.5 & 102.5 & - & & 74.5 & 0.5 & & \\
\hline & 84 & & & & 108.4 & & & & \\
\hline & 82 & & & & 127 & & & & \\
\hline 6 & 81.4 & 82.4 & 133 & & & & & & \\
\hline 7 & 80 & 81 & 143 & 1 & 143.5 & & & & \\
\hline $8^{*}$ & 77.6 & 78.75 & 216 & - & & 143 & - & & \\
\hline $9^{*}$ & 77.6 & 78.75 & 321 & - & & 211 & - & 143.5 & - \\
\hline 10 & 73.5 & 74.6 & 317 & - & & 141 & - & & \\
\hline $11^{*}$ & 66.67 & 68.1 & 314 & - & & 215 & - & 140 & - \\
\hline $12^{*}$ & 66.67 & 68.1 & 321 & - & & 222 & 3.5 & 144 & - \\
\hline 13 & 66.67 & 68.1 & 321 & 3 & 319.5 & & & & \\
\hline 14 & 50 & 51.6 & 321 & 3 & & 220 & 8 & & \\
\hline $15^{*}$ & 25 & 26.2 & 317.5 & - & & 226 & 20 & 134 & 1 \\
\hline 16 & 10 & 10.7 & 310 & - & & 217 & $\mathbf{3}$ & & \\
\hline 17 & 3.95 & 4.15 & $?$ & & & 230 & 11 & & \\
\hline 18 & 0 & 0 & 232 & & & & & & \\
\hline
\end{tabular}

punktserniedrigungen ron $\mathrm{Sn}$ und $\mathrm{SnJ}_{4}$ in Jod bis zu Konzertrationen von $1 \%$ bestimmt hat, findet normale Depressionen. In bezug auf den Erstarrungspunkt von $\mathrm{SnJ}_{2}$ sind die Werte von REINDERS und DE LANGE $\left(319-320^{\circ}\right)$ gleichfalls identisch mit den meinigen $(321 \%$.

Ein wesentlicher Unterschied aber liegt in der Darstellung des Dijodids. Erwärmt man nämlich (Versuch 8) vorsichtig $2.125 \mathrm{~g} \mathrm{Jod} \mathrm{mit}$ $7.875 \mathrm{~g}$ Zinn in einer zugeschmolzenen Glasröhre, so tritt bei etwa $50^{\circ}$ eine Reaktion ein, wobei die Temperatur bis etwa $250^{\circ}$ steigt. 


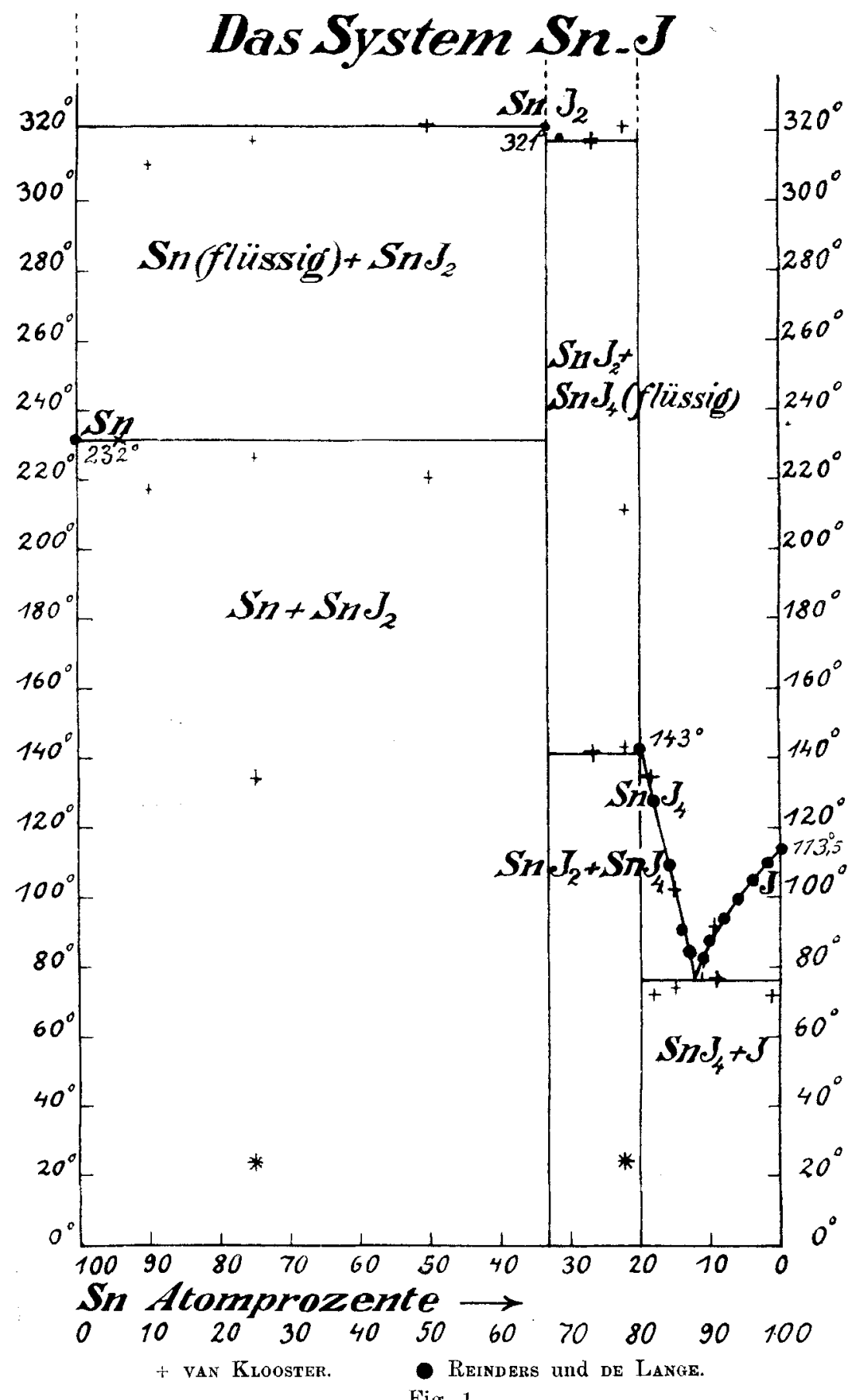

Fig. 1. 
Wird die Temperatur dann ungefähr während einer Stunde auf 230 bis $250^{\circ}$ gehalten, so findet man bei der nachfolgenden Abkühlung zwei Effekte, und es hinterbleibt $0.280 \mathrm{~g}$ Zinn in freiem Zustande. Dies entspricht einer Bildung von $\mathrm{SnJ}_{4}$ ohne merkliche Spuren von $\mathrm{SnJ}_{2}$. Dieses Resultat deckt sich somit vollkommen mit den Angaben Personnes. ${ }^{1}$ Wird dagegen eine Mischung derselben $\mathrm{Zu}$ sammensetzung bis auf etwa $400^{\circ}$ erhitzt, so bleibt $0.2 \mathrm{~g}$ Zinn ungelöst zurück neben $\mathrm{SnJ}_{2}$ und $\mathrm{SnJ}_{4}$. Die Abkühlungskurve zeigt drei Effekte, in Zusammenhang mit den Erstarrungspunkten des $\mathrm{SnJ}_{2}$ (mittlere Schicht), $\mathrm{Sn}$ und $\mathrm{SnJ}_{4}$ (obere Schicht). Auch diese Erscheinung ist von älteren Autoren wiederholt beobachtet worden. Es besteht aber auch hier kein Gleichgewicht, wie sich ergab bei den Versuchen zur Reindarstellung des $\mathbf{S n J} J_{2}$, frei von $\mathbf{S n}$ und $\mathrm{SnJ}_{4}$.

Nachdem zweimal versucht war eine Mischung ron $7.68 \mathrm{~g}$ Sn und $16.4 \mathrm{~g} \mathrm{~J}$ in geschlossener Röhre nach Behandlung im Schüttelapparat während etwa einer halben Stunde ( $t$ ca. $350^{\circ}$ ) homogen zu machen, zeigten doch jedesmal die Abkühlungskurven drei Effekte, trotzdem die Schmelze im Nickelofen das zweite Mal bis über $400^{\circ}$ erhitzt wurde. Die wechselnden Mengen unverbundenes Zinn, das eine Mal $2.5 \mathrm{~g}$, im zweiten Fall $2.8 \mathrm{~g}$ veranlaBten mich, nochmals eine gleiche Menge derselben Zusammensetzung (Versuch 13) im Schüttelapparat 3 Stunden auf $420-440^{\circ}$ zu exponieren. Die Abkühlungskurve zeigte dann nur einen Effekt bei $321^{\circ}$ und beim Öffnen der Röhre war nur noch eine minimale Zinnmenge (etwa 0.01 g) vorhanden. Vergleicht man dieses Resultat mit dem Experiment von Reinders und de Lange (1. e. S. 336), die aus $12.5 \mathrm{~g} \mathrm{SnJ}_{4}$; $7.5 \mathrm{~g} \mathrm{SnJ}_{2}$ und $2.5 \mathrm{~g} \mathrm{Sn} \mathrm{nach} 14$ stündiger Erhitzung auf $360^{\circ}$, $9.6 \mathrm{~g} \mathrm{SnJ}_{4}, 10.5 \mathrm{~g} \mathrm{SnJ}_{2}$ und $1.6 \mathrm{~g} \mathrm{Sn}$ erhielten, so wird hiermit in frappanter Weise der beschleunigende Einfluß erhöhter Temperatur auf das Zustandekommen der Reaktion $\mathrm{SnJ}_{4}+\mathrm{Sn}=2 \mathrm{SnJ}_{2}$ demonstriert. Auch wenn man ÜberschuB von Zinn anwendet, entsteht nur SnJ $J_{2}$ falls man lange und hoch genug erhitzt. Charakteristisch in dieser Beziehung ist Versuch 14, wobei $11.9 \mathrm{~g}$ Sn und $12.7 \mathrm{~g} \mathrm{~J}$ 3 Stunden auf etwa $400^{\circ}$ erhitzt wurden, wobei vollständige Gleichgewichtseinstellung eintrat; der primäre Effekt erschien ungeschwächt bei $321^{\circ}$ und beim Öffnen der Röhre waren genau $5.95 \mathrm{~g}$ Sn unverbunden zurückgeblieben. Der Versuch lehrt gleichfalls, daß Sn

1 Compt. rend. 54 (1862), 216. 
und $\mathrm{SnJ} \mathrm{J}_{2}$ sich bei Temperaturen von etwa $400^{\circ}$ nicht merkbar mischen, was die Angaben von ReINDERs und DE LANGE somit bestätigt.

Sodann wurde untersucht wie sich $\mathrm{SnJ}_{2}$ und $\mathrm{SnJ}_{4}$ in geschmolzenem Zustand nebeneinander erhalten.

$\mathrm{Zu}$ dem Zweck wurden $5.007 \mathrm{~g} \mathrm{SnJ}_{2}$ und $4.998 \mathrm{~g} \mathrm{SnJ}_{4}$ (Versuch 10) eine Stunde im Schüttelapparat auf etwa $360^{\circ}$ exponiert, und darauf im Nickelofen abkühlen gelassen. Es wurde einerseits eine Erniedrigung von $4^{\circ}$, andererseits von $2^{\circ}$ konstatiert. Eine Analyse der beiden erstarrten Schichten (versetzt mit $\mathrm{H}_{2} \mathrm{SO}_{4}$ s. g. 1.4, das Jod mittels $\mathrm{KNO}_{2}$ ausgetrieben ${ }^{1}$ und in der klaren Lösung das Zinn nach TreadweLL II, S. 193, bestimmt) lieferte für die SnJ $_{2}$-Schicht $31.1 \% \mathrm{Sn}$, für die $\mathrm{SnJ}_{4}-\mathrm{Schicht} 20.5 \% \mathrm{Sn} ; \mathrm{SnJ}_{2}$ und $\mathrm{SnJ}_{4}$ erfordern bez. $31.9 \%$ und $19 \%$ Sn. Reinders und de Lange (l. c. S. 338) finden $31.1 \%$ und $19 \%$. Hier liegt also eine kleine Differenz vor.

SchlieBlich wurden einige physische und kristallographische Konstanten an den $\mathrm{SnJ}_{4}$ - und $\mathrm{SnJ}_{2}$-Präparaten ermittelt.

Das $\mathrm{SnJ}_{2}$, dessen spezifisches Gewicht von BöDEKER ${ }^{2}$ auf 4.696 ermittelt wurde, und später von $\operatorname{RETGERS}^{3}(d=4.7)$, kristallisiert, wie schon von Groth, ${ }^{4}$ NordenskiöLd ${ }^{5}$ und Rergers (l. c.) konstatiert wurde, aus Lösungsmitteln in braunroten regulären Kristallen von vorwiegend oktaedrischem, bisweilen dodekaedrischen Habitus. Das geschmolzene Produkt zeigt eine etwas dunklere (kirschrote) Farbe, doch sind die optischen Eigenschaften dieselben, auch der Strich ist derselbe, nämlich rein kadmiumgelb.

Das $\mathrm{SnJ}_{2}$ kristallisiert in prächtig rubinroten Nadeln. In organischen Lösungsmitteln ist es, wenn nicht unter Jodabscheidung zersetzt, nur wenig löslich. Das spez. Gew., bestimmt in Paraffinöl, das zuvor mit $\mathrm{SnJ}_{2}$ gesättigt wurde, betrug: $d_{15}^{15}=5.21^{3}$. Das Pulver, sowie der Strich ist ziegelrot. Die Verbindung schmilzt, im offenen Rohr erhitzt, unzersetzt; für eine etwaige Umwandlung, die CоHEN ${ }^{6}$ auf Grund älterer Untersuchungen vermutete, konnte weder thermisch,

${ }^{1}$ Eine Analyse des Jods durch Auffangen in $\mathrm{Na}_{2} \mathrm{SO}_{3}$-Lösung und nach folgendem Zusatz von $\mathrm{Na}_{2} \mathrm{SO}_{3}, \mathrm{AgNO}_{3}$ und starker $\mathrm{HNO}_{3}$, wie Herr MENKE (Z. anorg. Chem. 77) angab für Jod-Bestimmung in T'e-J-Mischungen, ist nicht zulässig. Es wird dabei immer, wie man sich leicht an einem blinden Versuch überzeugen kann, mehr oder weniger $\mathrm{Ag}_{2} \mathrm{SO}_{4}$ mit ausgefüllt.

${ }^{2}$ Die Beziehung zwischen Dichte und Zusammensetzung, Leipzig 1860.

${ }^{3}$ Z. anorg. Chem. 3 (1893), 343; Zeitschr. f. Kryst. 22 (1894), 27 i.

${ }^{4}$ Chem. Krystallographie I, S. 231.

5 Bihang. K. Sv. Vet. Akad. Handl. 2 (1874), No. 2. 12.

${ }^{6}$ Abegas Handbuch III 2, S. 572. 
noch optisch eine Andeutung gefunden werden. Dagegen bestätigte sich die von Grotr (l. c. S. 208) geäuBerte Vermutung, daB die von NordensriöLd (l. c.) gemessenen rhombischen Kristalle (dargestellt durch Erhitzen von $\mathrm{Sn}$ in $\mathrm{HJ}$ und nachfolgender Erhitzung im geschlossenen Rohre) nicht der Formel $\mathrm{SnJ}_{2}$ entsprechen. Die kleinen nadelförmigen Kristalle von $\mathrm{SnJ}_{2}$, welche beim Erhitzen in vacuo durch Sublimierung erhalten wurden, konnten in der Prismenzone durchgemessen werden.

Die Messung (von Prof. JAEGER) ergab folgende Resultate:

Tabelle 2.

$\mathrm{SnJ}_{\mathbf{2}}$ : monoklin - prismatisch.

\begin{tabular}{|c|c|c|}
\hline & Gemessen & Berechnet \\
\hline $\begin{array}{l}(001):(101) \\
(101):(301) \\
(301):(100) \\
(100):(10 \overline{1}) \\
(10 \overline{1}):(00 \overline{1}) \\
(100):(00 \overline{1})\end{array}$ & $\begin{array}{l}* 28^{\circ} 40^{\prime} \\
26^{\circ} 36^{\prime} \\
27^{\circ} 39^{\prime} \\
\text { ca. } 65^{\circ} \\
\text { ca. } 32^{\circ} \\
{ }^{*} 97^{\circ} 5^{\prime}\end{array}$ & $\begin{array}{c}-\overline{ } \\
26^{\circ} 42^{\prime} \\
27^{\circ} 33^{\prime} \\
64^{\circ} 11^{\prime} \\
32^{\circ} 54^{\prime}\end{array}$ \\
\hline & $\begin{array}{l}\beta=82^{\circ} 55^{\prime \prime} \\
c: a=0.5911: 1\end{array}$ & \\
\hline
\end{tabular}

Optisch zeigten die Kristalle sich schwach pleochroitisch, nämlich orangeund rotbraun, und zwar rotbraun für Strahlen parallel der Orthodiagonale. Die optische Achsenebene ist $\{010\}$.

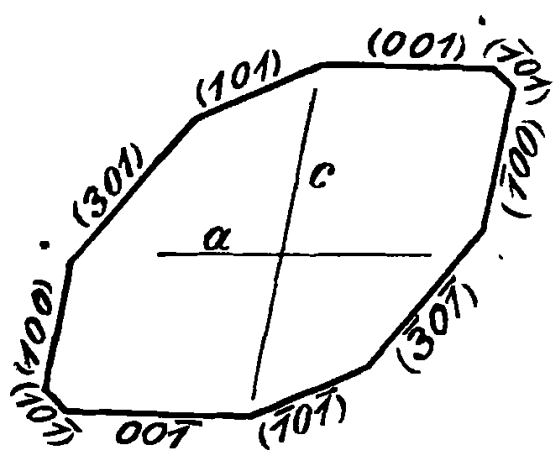

Fig. 2.

Groningen, Anorg.-cheri. Laboratorium der Reichsuniversität.

Bei der Redaktion eingegangen am 1. November 1912. 